\title{
More Practical Privacy-Preserving Machine Learning as A Service via Efficient Secure Matrix Multiplication
}

\author{
Wen-jie Lu \\ riku@mdl.cs.tsukuba.ac.jp \\ University of Tsukuba \\ Tsukuba, Ibaraki, Japan
}

\author{
Jun Sakuma \\ jun@cs.tsukuba.ac.jp \\ University of Tsukuba \\ Japan Science and Technology/CREST \\ RIKEN API Center \\ Tsukuba, Ibaraki, Japan
}

\begin{abstract}
An efficient secure two-party computation protocol of matrix multiplication allows privacy-preserving cloud-aid machine learning services such as face recognition and traffic-aware navigation. We use homomorphic encryption to construct a secure matrix multiplication protocol with a small communication overhead and computation overhead on the client's side, which works particularly well when a large number of clients access to the server simultaneously.

The fastest secure matrix multiplication protocols have been constructed using tools such as oblivious transfer, but a potential limitation of these methods is the needs of using a wide network bandwidth between the client and the server, e.g., $10 \mathrm{Gbps}$. This is of particular concern when thousands of clients interact with the server concurrently. Under this setting, the performance oblivious transfer-based methods will decrease significantly, since the server can only allocate a small ratio of its outgoing bandwidth for each client.

With three proposed optimizations, our matrix multiplication protocol can run very fast even under the high concurrent setting. Our benchmarks show that it takes an Amazon instance (i.e., 72 CPUs and 25 Gbps outgoing bandwidth) less than 50 seconds to complete 1000 concurrent secure matrix multiplications with $128 \times$ 128 entries. In addition, our method reduces more than $74 \%-97 \%$ of the precomputation time of two privacy-preserving machine learning frameworks, SecureML (S\&P'17) and MiniONN (CCS’17).
\end{abstract}

\section{CCS CONCEPTS}

\section{- Security and privacy $\rightarrow$ Privacy-preserving protocols;}

\section{KEYWORDS}

Secure Two-party Computation; Multiplication Triple; Homomorphic Encryption; Privacy-Preserving Machine Learning

\section{ACM Reference Format:}

Wen-jie Lu and Jun Sakuma. 2018. More Practical Privacy-Preserving Machine Learning as A Service via Efficient Secure Matrix Multiplication. In

Permission to make digital or hard copies of all or part of this work for personal or classroom use is granted without fee provided that copies are not made or distributed for profit or commercial advantage and that copies bear this notice and the full citation on the first page. Copyrights for components of this work owned by others than ACM must be honored. Abstracting with credit is permitted. To copy otherwise, or republish, to post on servers or to redistribute to lists, requires prior specific permission and/or a fee. Request permissions from permissions@acm.org.

WAHC '18, October 19, 2018, Toronto, ON, Canada

(C) 2018 Association for Computing Machinery.

ACM ISBN 978-1-4503-5987-0/18/10 ..\$15.0

https://doi.org/10.1145/3267973.3267976 6th Workshop on Encrypted Computing Applied Homomorphic Cryptography (WAHC '18), October 19, 2018, Toronto, ON, Canada. ACM, New York, NY, USA, 12 pages. https://doi.org/10.1145/3267973.3267976

\section{INTRODUCTION}

Machine learning is becoming ubiquitous. More and more machine learning-based online services, such as shopping recommendation [30], traffic-aware navigation [38], medical diagnosis [33], and face recognition [11], are reachable by thousands of clients. One important feature of these online services is high concurrency. That is, there might be thousands of clients using the services simultaneously. Suppose a traffic-aware navigation service is running in a metropolitan area, e.g., Tokyo and New York. The number of clients using the navigation service can be numerous during the peak time. Thus, the machine learning-based service should be scalable under this highly concurrent setting. Moreover, clients of these online services are usually equipped with limited computing resources. Take the navigation service as the example again; the client might connect to the navigation service via his/her cell phone or through on-vehicle devices. Therefore, the machine learning-based service should avoid introducing heavy computations at the client's side.

To use a machine learning-based online service, a client must reveal his/her data to the server. When the data involves sensitive information of the client, such as locations, shopping logs and medical records, revealing these data to the server might raise potential risks of compromising client's privacy, such as data breaches. A natural question here is whether one can use the machine learningbased online services and still maintain the privacy of client's data?

One solution is to use secure two-party computation (2PC) techniques [17, 39]. 2PC allows two parties (e.g., the client and the server) to jointly compute a function on their private inputs, learning only the output of the function. In the context of the privacypreserving machine learning, one of the most fundamental components is a practical secure matrix multiplication protocol, since computing the product of matrices is the essential operation of many popularly used machine learning algorithms such as linear regression [13, 24], logistic regression [36] and neural networks [23, 31].

Based on the discussions above, the main objective of this work is to develop a practical secure matrix multiplication protocol that can run fast under the following (realistic) situations.

(1) High Concurrency. There can be thousands of clients continuously and concurrently access to the server while the outgoing and incoming bandwidth of the server and clients are bounded, e.g., $20 \mathrm{Gbps}$ and $100 \mathrm{Mbps}$, respectively. In 


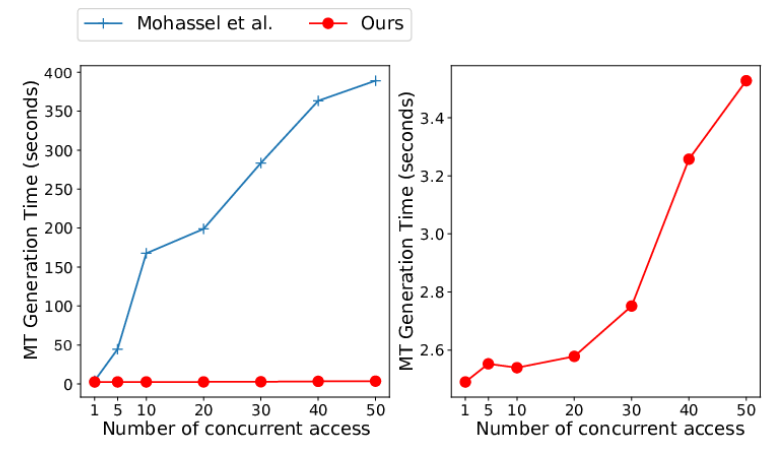

Figure 1: Left: Running time for generating MTs for multiplying matrices of $128 \times 128$ entries using the OT-based method of Mohassel et al. [28] under a 25 Gbps outgoing bandwidth. Right: Running time of our method under the same setting.

other words, the server can only allocate a small ratio of its bandwidth for each client.

(2) Weak Client. The computing resources at the client's side might be constrained. For example, the computing power of the autonomous vehicles and the Internet of Things (IoT) devices are usually limited, e.g., one 1.0 - 2.0 GHz CPU chip.

\subsection{Related Work and Challenges}

Recent improvements and optimizations to $2 \mathrm{PC}$, such as $[2,4,8,9$, $19,22,28,35,37]$ to name a few, enable efficient secure matrix multiplication protocols. Specifically, we can separate a secure multiplication protocol into two stages: an offline stage and an online stage. The computation during the offline stage is input-independent, that is the client and the server do not need to provide any private data in this stage. They jointly compute some (one-time use) auxiliary data, i.e., Beaver's multiplication triples (MTs) [3], so that the evaluation of the online stage can be significantly accelerated using MTs.

Beaver's MT is one of the most efficient way to perform secure matrix multiplication which has been applied to many $2 \mathrm{PC}$ frameworks such as SPDZ [8], ABY [9], SecureML [28] and MiniONN [23]. Many optimizations are proposed to improve the MT generation (i.e., the performance in the offline stage) in these frameworks. Specifically, ABY and SecureML suggest to use a vectorization technique and propose a variant type of MT which is specialized for secure dot product. Then, secure matrix multiplication can be computed by the iterations of secure dot products. They present two concrete solutions from oblivious transfer (OT) and additively homomorphic encryption (AHE), respectively. On the other hand, SPDZ and MiniONN propose another optimization from a somewhat homomorphic encryption (SwHE) scheme $[4,6]$ and the message packing technique [34]. Specifically, a batch of $\ell>0$ integers are encrypted as a single ciphertext where $\ell$ is a parameter related to the SwHE scheme. Then, the homomorphic multiplication will be carried out to the packed $\ell$ integers simultaneously.

The secure matrix multiplication protocol can also be constructed from tools such as Yao's garbled circuit [20, 30, 39, 41] and garbled arithmetic circuit [1]. However, such generic tools also require a wide bandwidth as the OT-based methods. Other ad hoc methods $[10,27]$ are efficient regarding computation time but the matrix size is constrained, e.g., smaller than $16 \times 16$, which might not be sufficient for modern machine learning algorithms like deep neural networks.

Challenges. Suppose the client and the server evaluate a secure matrix multiplication with private matrices of $n$ dimension of $t$-bits integers using the existing methods $[8,9,23,28]$. We now show that these methods might not scale well under the high concurrency and weak client situations.

For the OT-based method of $[9,28]$, the client and the server need to perform $O\left(n^{3} t\right)$ instances of correlated OT [2], which is lightweight in terms of computation. However, when many clients access to the server concurrently, these OT-based methods will take a considerably longer time, since the bandwidth allocated for each user is eventually bounded. Figure 1 gives an example of using the OT-based method of [28] to generate MTs for secure matrix multiplication of two $128 \times 128$ matrices. In this experiment, the outgoing bandwidth on the server's side was about $25 \mathrm{Gbps}$. When the number of concurrent accesses was small, e.g., $1-2$, clients only need to wait less than 5 seconds. As the number of concurrent accesses increases, the waiting time becomes significantly longer. In other words, if the server wants to handle 1000 concurrent accesses within 5 seconds, it might need to prepare more than 25000 Gbps outgoing bandwidth, which seems unrealistic for the current Internet.

For the SwHE-based method of [23], the client and the server exchange $O\left(n^{3} / \ell\right)$ SwHE ciphertexts through the network. This method can consume less bandwidth than the OT-based solutions because of the packing technique allows embedding a large number of plaintext values into a single ciphertext, such as $\ell=2^{12}$. However, this method requires the client to perform $O\left(n^{3} / \ell\right)$ unpackings, which can be extremely expensive for a weak client. For instance, in our benchmarks, it took a Raspberry Pi more than 23 minutes to perform the unpacking when $n=128$ and $\ell=2^{12}$. Even for a powerful AWS instance, this computation still took more than 2 minutes.

For the AHE-based method of $[9,28]$, the client and the server exchange $O\left(n^{2}\right)$ AHE ciphertexts. The client needs to perform $O\left(n^{2}\right)$ decryptions, and no unpacking is needed. It seems that the AHEbased method is the best under our situations. However, the server needs to operate $O\left(n^{3}\right)$ public key operations which can become the performance bottleneck when $n$ is large. For instance, when $n=512$, the client would need to wait more than 6 minutes before the server completes the secure matrix multiplication.

\subsection{Our Results and Contributions}

In this work, we present a more practical secure matrix multiplication protocol which can also work well under the high concurrency and weak client setting without assuming any trusted third party. Speaking from one of the experiment results, i.e., right-hand-side of Figure 1, to handle 50 concurrent accesses, it just took less than 4 seconds, which significantly outperformed the OT-based method [28]. In $\S 6.5$, we further benchmarked our method with 1000 concurrent accesses and demonstrated that it took less than 1 minute to 
Table 1: Comparing the computation complexity of three HE-based methods. $m$ and $\ell$ are parameters of the underlying SwHE and RLWE-based scheme.

\begin{tabular}{c|cc}
\hline Method & Client & Server \\
\hline AHE-based [28] & $O\left(n^{2}\right)$ & $O\left(n^{3}\right)$ \\
SwHE-based [23] & $O\left(n^{3} / m\right)$ & $O\left(n^{3} / m\right)$ \\
RLWE-basd Ours & $O\left(n^{2} / \ell\right)$ & $O\left(n^{3} / m\right)$ \\
\hline
\end{tabular}

complete all the computation. We also conducted experiments on a weak device, i.e., Raspberry Pi, and we verified that our method can also perform well for relatively weak devices. For example, Raspberry Pi took about 17 seconds to complete the secure matrix multiplication of a matrix in the size of $128 \times 128$. We applied our method to two privacy-preserving machine learning frameworks, i.e., SecureML [28] and MiniONN [23]. Experimental results show that our method reduced about $74 \%-97 \%$ of the MT generation time of them (§ 7).

Our Technical Contributions. The technical contributions of this work are summarized as follows.

- Overall, we present a simple and extremely fast secure matrix multiplication protocol (§5). Our method is built from ring learning with error (RLWE)-based homomorphic encryption and it outperforms the state-of-the-art methods under the high concurrency and weak client setting.

- We propose new optimizations (\$4) which allow us to homomorphically compute a batch of $\ell$ inner products at the cost of a single homomorphic operation. The server only needs to operate $O\left(n^{3} / m\right)$ homomorphic operations, where $m$ is usually large for example $m \geq 2^{12}$. The client only performs $O\left(n^{2} / \ell\right)$ decryptions and unpackings. The comparison to existing methods is summarized in Table 1.

- We also propose an implementation optimization (\$4.4) to accelerate the computation at the client's side. Specifically, we use a pre-computed table which can be constructed from the client's public parameters. Therefore, this table can be used by the client for multiple times once it is computed. This optimization significantly reduces the client's workload, which is especially important for a weak client. According to our experiment results, this pre-computed table reduces about $60 \%$ computation time at the client's side at the cost of a negligible pre-computation effort.

Roadmap. $\S 2$ introduces problem statements, security model and the underlying RLWE-based encryption scheme used our construction. After presenting a basic secure matrix multiplication protocol in $\S 3$, the proposed optimizations to the basic protocol are presented in $\S 4$. Then, the full protocol is given in 5 . Evaluation results are given in $\S 6$. The comparison results to MiniONN and SecureML are given in $\S 7$. Then, $\S 8$ concludes the paper.

\section{PRELIMINARIES}

Table 2 summarizes the notation that used in this paper. Typically, we write $[\ell]$ to denote the set of positive integers $\{0,1, \ldots, \ell-$ $1\}$, and write the upper case Roman character $X$ to denote the determinant of polynomials.
Table 2: Notation

\begin{tabular}{c|l}
\hline$[\ell]$ & set of positive integers $\{0,1, \ldots, \ell-1\}$ \\
$r \stackrel{\$}{\leftarrow} \mathcal{E}$ & uniformly sample $r$ at random from $\mathcal{E}$ \\
$\mathrm{a}, a_{i}$ & vector and its $i$-th entry \\
$\mathbf{U}, \mathbf{U}[i, j]$ & matrix and its $(i, j)$-th entry \\
$\mathbf{U}[i,:], \mathbf{U}[, j]$ & the $i$-th row and $j$-th column of $\mathbf{U}$ \\
$P, P[i]$ & polynomial and its $i$-th coefficient \\
$X$ & determinant of polynomials \\
$\langle a\rangle_{0},\langle a\rangle_{1}$ & additive share of integer $a$ \\
$\langle\mathrm{~A}\rangle_{0},\langle\mathrm{~A}\rangle_{1}$ & additive share of matrix A \\
$\mathbb{R}_{\boldsymbol{q}}$ & plaintext space $\mathbb{R}_{\boldsymbol{q}}:=\mathbb{Z}_{\boldsymbol{q}}[X] /\left(X^{m}+1\right)$ \\
\hline
\end{tabular}

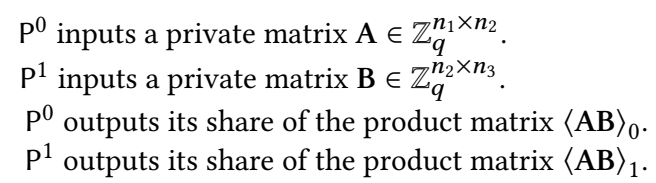

Figure 2: Ideal functionality $\mathcal{F}_{\mathrm{MP}}$ for matrix product.

\subsection{Problem Statements}

In this work, we focus on protocols with two players, $\mathrm{P}^{0}$ (e.g., the client) and $\mathrm{P}^{1}$ (e.g., the server)

We consider the functionality $\mathcal{F}_{M P}$ of Figure 2, where A and B is the private input (not additive shares) of $\mathrm{P}^{0}$ and $\mathrm{P}^{1}$, respectively. Specifically, we present a $2 \mathrm{PC}$ protocol SMP.

$$
\operatorname{SMP}(A, B) \rightarrow\left(\langle A B\rangle_{0},\langle A B\rangle_{1}\right) .
$$

In other words, SMP takes as of two private matrices and outputs additive shares of the multiplication result.

\subsection{Security Model}

We use the semi-honest adversary model. That is, $\mathrm{P}^{0}$ and $\mathrm{P}^{1}$ follow the protocol specification but might attempt to learn more information via the protocol communication.

Our security definitions follow the real-world/ideal-world paradigm of $[5,16]$. Specifically, we compare the protocol execution in the real world to an execution in the ideal world. In the real world, protocol players follow the specification of a protocol $\Pi$, and in the ideal world, protocol players have access to a trusted third party (TTP) that evaluates the functionality $\mathcal{F}$. The protocol execution is viewed as occurring in the existence of an adversary $\mathcal{A}$ and cooperated with an environment $C=\left\{C_{K}\right\}$ which is modeled as a class of polynomial-size circuits parameterized by a security parameter $\kappa$. The role of the environment is to choose the input to the protocol execution and to distinguish experiments in the real world and the ideal world. Informally, a protocol $\Pi$ privately implements $\mathcal{F}$ if the adversary $\mathcal{A}$ can not learn anything about the inputs of the other protocol player beyond what is explicitly revealed by the outputs of the computation.

\subsection{Ring-based Homomorphic Encryption}

We use a ring learning with errors [26] based homomorphic encryption for which plaintext space is defined as the quotient ring 
$\mathbb{R}_{q}=\mathbb{Z}_{q}[X] /\left(X^{m}+1\right)$ where $m$ is power of 2 and $q$ is a prime number. Many existing encryption schemes can be used in our construction, such as BGV's leveled homomorphic encryption scheme [4] and FV's SwHE scheme [12]. Typically, we use the symmetric version of the BGV scheme because of the most functioning implementation, i.e., HElib [32].

We give notations of functions of the underlying encryption scheme but leave the details to their paper [4]. The BGV scheme consists of five functions $\{$ KeyGen, Enc, Dec, Add, Mult $\}$. KeyGen takes as input a security parameter $\kappa$ and outputs a private key sk and an evaluation key evk. The encryption of a plaintext $A \in \mathbb{R}_{q}$ is written as $\llbracket A \rrbracket=\operatorname{Enc}(A ; \mathrm{sk})$, and the decryption is denoted as $\operatorname{Dec}(\llbracket A \rrbracket ; \mathrm{sk})$. The private key sk in the encryption and decryption functions will be omitted if sk is inferred clearly from the context.

We can perform addition of ciphertexts and multiplication of ciphertext and scalar using the evaluation key evk. Suppose $A, B \in$ $\mathbb{R}_{q}$ are two plaintext values.

- Homomorphic addition of two ciphertexts

$$
\operatorname{Dec}(\operatorname{Add}(\operatorname{Enc}(A), \operatorname{Enc}(B) ; \operatorname{evk}))=A+B \text {. }
$$

- Homomorphic addition and multiplication between a ciphertext and a plaintext

$$
\begin{aligned}
\operatorname{Dec}(\operatorname{Add}(\operatorname{Enc}(A), B ; \operatorname{evk})) & =A+B, \\
\operatorname{Dec}(\operatorname{Mult}(\operatorname{Enc}(A), B ; \operatorname{evk})) & =A \times B .
\end{aligned}
$$

Simply, we write $\llbracket A \rrbracket \oplus \llbracket B \rrbracket$ and $\llbracket A \rrbracket \otimes B$ to indicate the homomorphic addition and homomorphic multiplication, respectively. Furthermore, we write $\ominus$ to denote the homomorphic subtraction.

\subsection{Message Packing}

Message packing techniques are powerful tools to reduce the spatial and computational overhead of homomorphic encryption based secure protocols, e.g., [14, 15, 24, 25, 29]. Generally, multiple messages are encrypted as a single ciphertext by the virtue of the algebraic structure of $\mathbb{R}_{q}$. Therefore, fewer ciphertexts are generated. More importantly, various types of computation can be operated efficiently, according to the packing method.

The Chinese-Remainder-Theorem (CRT) packing presented in [34] is the most commonly used packing technique. This technique decomposes the plaintext space $\mathbb{R}_{q}$ into $\ell$ plaintext slots, allowing $\ell$ messages to be encrypted as a single ciphertext. Then homomorphic operations on the packed ciphertexts will be carried to each slot simultaneously, reducing the time of homomorphic computation by a factor of $\ell$. Specifically, the polynomial $X^{m}+1$ is factorized into $\ell$ distinct and degree- $d$ factor polynomials $F_{k}$ such that

$$
X^{m}+1=\prod_{k=0}^{\ell-1} F_{k} \quad \bmod q, \text { and } m=d \cdot \ell .
$$

From the Chinese Remainder Theorem, the plaintext space $\mathbb{R}_{q}$ is isomorphic to the product of $\ell$ sub-spaces, i.e., $\mathbb{R}_{q} \cong \prod_{j=0}^{\ell-1} \mathbb{Z}_{q}[X] /\left(F_{k}\right)$. It is noteworthy that the sub-space $\mathbb{Z}_{q}[X] /\left(F_{k}\right)$ is usually viewed as the vector space $\mathbb{Z}_{q}^{d}$. However, in $\S 4.2$, we examine the property of the polynomial $F_{k}$, and thus we keep using the polynomial view.

We write $\pi_{\text {crt }}: \prod_{j=0}^{\ell-1} \mathbb{Z}_{q}[X] /\left(F_{k}\right) \mapsto \mathbb{R}_{q}$ to denote the CRTpacking that converts $\ell$ polynomials to an element of $\mathbb{R}_{q}$, and write
-Input of the client: private matrix $A \in \mathbb{Z}_{q}^{n_{1} \times n_{2}}$

-Input of the server: private matrix $\mathrm{B} \in \mathbb{Z}_{q}^{n_{2} \times n_{3}}$

-Output of the client: additively shared matrix $\langle\mathrm{AB}\rangle_{0}$

-Output of the server: additively shared matrix $\langle A B\rangle_{1}$

1: Encryption. The client encrypts the elements of $\mathbf{A}$ and sends $n_{1} n_{2}$ ciphertexts $\left\{\operatorname{Enc}\left(a_{i k}\right)\right\}_{0 \leq i<n_{1}, 0 \leq k<n_{2}}$ to the server.

2: Evaluation. The server

(1) samples a random matrix $\mathrm{C}^{1} \stackrel{\$}{\leftarrow} \mathbb{Z}_{q}^{n_{1} \times n_{3}}$.

(2) computes the matrix multiplication via homomorphic operations. For example, the $(i, j)$-th entry of the product matrix is computed by

$$
\operatorname{Enc}\left(c_{i j}^{0}\right)=\left(\bigoplus_{k=0}^{n_{2}-1} \operatorname{Enc}\left(a_{i k}\right) \otimes b_{k j}\right) \ominus c_{i j}^{1} .
$$

(3) returns $n_{1} n_{3}$ ciphertexts $\left\{\operatorname{Enc}\left(c_{i j}^{0}\right)\right\}_{0 \leq i<n_{1}, 0 \leq j<n_{3}}$ to the client.

(4) outputs the matrix $\mathrm{C}^{1}$.

3: Extraction. The client decrypts $\left\{\operatorname{Enc}\left(c_{i j}^{0}\right)\right\}$ and outputs the decrypted values in the form of a matrix $\mathrm{C}^{0}$.

\section{Figure 3: Basic secure matrix product protocol.}

$\pi_{\mathrm{crt}}^{-1}$ as the reversing function. Let $\mathbf{x}$ and $\mathbf{y}$ be vectors of $\ell$ polynomials, where $x_{k}, y_{k} \in \mathbb{Z}_{q}[X] /\left(F_{k}\right)$ for $k \in[\ell]$. Homomorphic additions and multiplications of CRT-packed ciphertexts are carried to each plaintext slot simultaneously. That is, from following computation

$$
\begin{aligned}
& \operatorname{Enc}\left(\pi_{\mathrm{crt}}(\mathbf{x})\right) \oplus \operatorname{Enc}\left(\pi_{\mathrm{crt}}(\mathbf{y})\right) \\
& \operatorname{Enc}\left(\pi_{\mathrm{crt}}(\mathbf{x})\right) \otimes \operatorname{Enc}\left(\pi_{\mathrm{crt}}(\mathbf{y})\right),
\end{aligned}
$$

we can obtain $\ell$ additions $\left\{x_{k}+y_{k} \bmod F_{k}\right\}_{k \in[\ell]}$, and $\ell$ products $\left\{x_{k} \times y_{k} \bmod F_{k}\right\}_{k \in[\ell]}$.

\section{THE BASIC PROTOCOL}

We give an informal description of our basic protocol in Figure 3 which follows the general framework of the existing homomorphic encryption-based methods such as $[8,9,15,23,28]$, i.e., computing inner products via homomorphic operations. In the methods of $[9,15,28]$, the client needs to perform $O\left(n_{1} n_{2}+n_{1} n_{3}\right)$ homomorphic operations and the server needs to perform $O\left(n_{1} n_{2} n_{3}\right)$ homomorphic operations. The optimization from $[8,23]$ reduces the number of operations on the server's side to $O\left(n_{1} n_{2} n_{3} / m\right)$ by using the CRT packing, at the cost of increasing the client's work to $O\left(n_{1} n_{2} n_{3} / m\right)$.

\section{PROPOSED OPTIMIZATIONS}

We present our new optimizations which reduce the number of operations at the server's side to $O\left(n_{1} n_{2} n_{3} / m\right)$ and that at the client's side to $O\left(n_{1} n_{2} / m+n_{1} n_{3} / \ell\right)$. 


\subsection{Forward-backward Encoding}

The basic protocol in Figure 3 requires the server to perform $O\left(n_{1} n_{2} n_{3}\right)$ homomorphic multiplications and additions due to the computation of inner products of vectors. Our first optimization forwardbackward (FB) encoding aims to improve the performance of computing inner products, which is inspired by $[25,40]$. Intuitively, when multiplying two general polynomials of degree- $\delta(\delta>0)$, the coefficient of $X^{\delta-1}$ in the product polynomial is the inner product of the two coefficient vectors in opposite orders. Specifically, we define two functions to convert vectors $\mathbf{u}, \mathbf{v} \in \mathbb{Z}_{q}^{\delta}$ to polynomials

$$
\vec{e}(\mathbf{u})=\sum_{i=0}^{\delta-1} u_{i} X^{i}, \quad \overleftarrow{e}(\mathbf{v})=\sum_{j=0}^{\delta-1} v_{j} X^{\delta-1-j}
$$

We write $\vec{e}$ and $\overleftarrow{e}$ as respectively representing the forward encoding and backward encoding. The inner product $\mathbf{u}^{\top} \mathbf{v}$ can be given by one polynomial multiplication as shown below.

$$
P[\delta-1]=\mathbf{u}^{\top} \mathbf{v}, \text { where } P=\vec{e}(\mathbf{u}) \times \overleftarrow{e}(\mathbf{v})
$$

The correctness of Equation 3 can be shown as

$$
P[\delta-1]=\sum_{i, j: i+\delta-1-j=\delta-1} u_{i} v_{j}=\sum_{i, j: i=j} u_{i} v_{j} .
$$

It is equal to $\mathbf{u}^{\top} \mathbf{v}$. This equation holds for any degree $\delta>0$. However, the ring-based encryption scheme can handle at most degree$m$ polynomials. Fortunately, we can use partitions for larger degree of polynomials. Suppose the length of vectors $\mathbf{u}^{\prime}$ and $\mathbf{v}^{\prime}$ is $K m$ for $K>0$, and the vectors are partitioned equally into $K$ pieces, i.e., $\mathbf{u}^{\prime}=\mathbf{u}_{0}^{\prime}\|\ldots\| \mathbf{u}_{K-1}^{\prime}$ and $\mathbf{v}^{\prime}=\mathbf{v}_{0}^{\prime}\|\ldots\| \mathbf{v}_{K-1}^{\prime}$ where $\cdot \| \cdot$ denotes vectors concatenation. It is easy to see that from $K$ polynomial multiplications and additions, i.e., $\sum_{k}^{K} \vec{e}\left(\mathbf{u}_{k}^{\prime}\right) \times \overleftarrow{e}\left(\mathbf{v}_{k}^{\prime}\right)$, we can obtain the inner product of the longer vectors, i.e., $\mathbf{u}^{\prime \top} \mathbf{v}^{\prime}$. To simplify the exposition, we write $\vec{e}\left(\mathbf{u}^{\prime}\right)$ and $\overleftarrow{e}\left(\mathbf{v}^{\prime}\right)$ implicitly denoting the encoding on the partitioned vectors.

We can apply FB-encoding to reduce both the computational and communication cost of the basic protocol as follows. The client processes each row of its private matrix A with $\vec{e}$ before the encryption, resulting in $n_{1} n_{2} / m$ ciphertexts, $\{\operatorname{Enc}(\vec{e}(\mathrm{~A}[i,:]))\}_{0 \leq i<n_{1}}$ which are sent to the server. Upon receiving each ciphertext, the server homomorphically computes the inner product $\operatorname{Enc}(\vec{e}(\mathrm{~A}[i,:])) \otimes \overleftarrow{e}(\mathrm{~B}[$ $, j]) \ominus c_{i j}^{1}$ for each column $0 \leq j<n_{3}$ and $c_{i j}^{1} \stackrel{\$}{\leftarrow} \mathbb{Z}_{q}$, and sends $n_{1} n_{3}$ ciphertexts back to the client. The FB-encoding technique enables the server to compute the matrix product via $n_{1} n_{2} n_{3} / m$ homomorphic operations. Since $m$ usually has size of few thousands, e.g., $m \geq 2^{12}$, this translates to a considerable improvement over the basic protocol.

\subsection{Double Packing}

With the FB-encoding technique of $\S 4.1$, we significantly reduce the computational cost at the server's side. However, the client still needs to decrypt $n_{1} n_{3}$ ciphertexts. It would be nice if we can even reduce the computational cost at the client's side, since the computing power of the client might be very limited. We will achieve this through a new optimization double packing which comes from an insightful observation on the CRT packing and the FB-encoding technique. Specifically, this optimization allows the server to compute a batch of $\ell>0$ inner products within one single ciphertext. The server now will send $n_{1} n_{3} / \ell$ ciphertexts to the client. Thus, this provides a $\ell$-fold improvement to the communication cost and computational cost at the client's side.

From a high-level explanation, the double packing employs the FB-encoding to each plaintext slot of the CRT packing. One should recall that the CRT packing introduces an isomorphic map $\mathbb{Z}_{q}[X] /\left(X^{m}+1\right) \cong \prod_{k=0}^{\ell-1} \mathbb{Z}_{q}[X] /\left(F_{k}\right)$, where the factor polynomial $F_{k}$ is a degree- $d$ polynomial and $m=d \ell$. In other words, the polynomial multiplication in each sub-field is over the modulo $F_{k}$. Without assuming the arrangement of $F_{k}$ s' coefficients, we have Theorem 4.1.

Theorem 4.1. Presume integer vectors $\mathbf{u}, \mathbf{v} \in \mathbb{Z}_{q}^{\delta}$. Let $F_{k}$ be one of the degree-d factor polynomial from the CRT-packing. If $0<\delta \leq d / 2$, then the following holds.

$$
P[\delta-1]=\mathbf{u}^{\top} \mathbf{v} \text {, where } P=\vec{e}(\mathbf{u}) \times \overleftarrow{e}(\mathbf{v}) \bmod F_{k}
$$

Proof. If the vector length $\delta \leq d / 2$, then the degree of the product polynomial $\vec{e}(\mathbf{u}) \times \overleftarrow{e}(\mathbf{v})$ is less than $d$. As a result, taking modulo of the degree- $d$ polynomial $F_{k}$ does not change the correctness of Equation 3.

Theorem 4.1 simply implies that a batch of $\ell$ inner products of vectors of length $d / 2$ can be homomorphically computed via one homomorphic multiplication, resulting in one single ciphertext. To simplify the exposition, we reform the batch of $\ell$ inner products as a matrix-vector product. Suppose the matrix $U \in \mathbb{Z}_{q}^{\ell \times d / 2}$ and the vector $\mathbf{v} \in \mathbb{Z}_{q}^{d / 2}$. To homomorphically compute the product $\mathrm{Uv}$, the row vectors of $U$ can be encrypted as one ciphertext ctx using the FB-encoding and CRT packing

$$
\operatorname{ctx}:=\operatorname{Enc}\left(\pi_{\mathrm{crt}}([\vec{e}(\mathbf{U}[0,:]), \ldots, \vec{e}(\mathrm{U}[\ell-1,:])])\right) .
$$

Similarly, the vector $\mathbf{v}$ is converted to one polynomial $V$

$$
V=\pi_{\mathrm{crt}}([\underbrace{\overleftarrow{e}(\mathbf{v}), \ldots, \overleftarrow{e}(\mathbf{v})}_{\ell \text { copies }}])
$$

From one homomorphic multiplication $\operatorname{ctx} \otimes V$, we can obtain the $\ell$ inner products of length $d / 2$ vectors, i.e., Uv.

When using the double packing for secure matrix multiplication, the client will break down each row of $A$ into vectors with at most $d / 2$ entries, and encrypt $\ell$ vectors that picked from distinct row as one ciphertext. In total, the client will send $n_{1} / \ell \cdot 2 n_{2} / d \approx 2 n_{1} n_{2} / m$ ciphertexts to the server. To compute the matrix product, the server first breaks down each column vector of $\mathbf{B}$ into vectors with at most $d / 2$ entries, and encodes $\ell$ copies of each shorter vector as one polynomial. Then, the server can homomorphically compute the matrix product via about $2 n_{1} n_{2} n_{3} / m$ operations, resulting in $n_{1} n_{3} / \ell$ ciphertexts. This means the decryptions at the client's side is reduced by $1 / \ell$ over the optimization in $\S 4.1$.

\subsection{Double the Capacity of Double Packing}

Using the optimization in $\S 4.2$, we can compute $\ell$ inner products of vectors with $d / 2$ entries. We now present a way to double this 
Table 3: Prime $q$ that satisfies Eq. 4 when $\mathrm{m}=4096$.

\begin{tabular}{c|cccccc}
\hline$q$ & 84961 & 82241 & 82561 & 70913 & 84481 & 87041 \\
\hline$\ell$ & 16 & 32 & 64 & 128 & 256 & 512 \\
\hline
\end{tabular}

length from $d / 2$ to $d$ which helps reducing half of the computation time and communication cost of our secure matrix product protocol. To do so, we must find a such prime $q$ that "shapes" all polynomials $F_{k}$ into a specific form

$$
F_{k}=X^{d}+\beta_{k} \text { s.t. } \beta_{k} \neq 0 .
$$

For example, polynomial $X^{1024}+1$ can be decomposed as $\left(X^{256}+\right.$ $10)\left(X^{256}+41\right)\left(X^{256}+96\right)\left(X^{256}+127\right) \bmod 137$, that is $m=1024$ $d=256$, and $q=137$. With such $F_{k}$ 's, we can apply Equation 3 to the plaintext slots with vectors of more than $d$ entries.

THEOREM 4.2. Suppose integer vectors $\mathbf{u}, \mathbf{v} \in \mathbb{Z}_{q}^{d}$ and $\beta_{k} \neq 0$. Then the following holds.

$$
P[d-1]=\mathbf{u}^{\top} \mathbf{v} \text {, where } P=\vec{e}(\mathbf{u}) \times \overleftarrow{e}(\mathbf{v}) \bmod X^{d}+\beta_{k}
$$

Proof. Before taking the modulo $X^{d}+\beta_{k}$, the $(d-1)$-th coefficient of the product $\vec{e}(\mathbf{u}) \times \overleftarrow{e}(\mathbf{v})$ equals to $\mathbf{u}^{\top} \mathbf{v}$, according to Equation 3. In addition, the degree of this polynomial is $2 d-2$. Therefore, the $(d-1)$-th coefficient remains unchanged, even taking the modulo $X^{d}+\beta_{k}$.

Similarly to Theorem 4.1, Theorem 4.2 implies that a batch of $\ell$ inner products of vectors of length $d$ can be homomorphically computed via one homomorphic multiplication, resulting in one single ciphertext if parameters $m$ and $q$ are chosen properly. This means the computational cost in $\S 4.2$ is reduced by a half. That is, the number of encryptions performed by the client is reduced from $2 n_{1} n_{2} / m$ to $n_{1} n_{2} / m$ and the number of homomorphic operations operated by the server is reduced from $2 n_{1} n_{2} n_{3} / m$ to $n_{1} n_{2} n_{3} / m$.

Finally, we write $\vec{\pi}_{\mathrm{w}}$ and $\overleftarrow{\pi}_{\mathrm{w}}:\left(\mathbb{Z}_{q}^{d}\right)^{\ell} \mapsto \mathbb{R}_{q}$ respectively denoting the double packing functions

$$
\begin{aligned}
& \vec{\pi}_{\mathrm{w}}\left(\mathbf{u}_{0}, \ldots, \mathbf{u}_{\ell-1}\right)=\pi_{\mathrm{crt}}\left(\vec{e}\left(\mathbf{u}_{0}\right), \ldots, \vec{e}\left(\mathbf{u}_{\ell-1}\right)\right) \\
& \overleftarrow{\pi}_{\mathrm{w}}\left(\mathbf{v}_{0}, \ldots, \mathbf{v}_{\ell-1}\right)=\pi_{\mathrm{crt}}\left(\overleftarrow{e}\left(\mathbf{v}_{0}\right), \ldots, \overleftarrow{e}\left(\mathbf{v}_{\ell-1}\right)\right)
\end{aligned}
$$

The subscript ' $w$ ' means double packing.

Parameter Choosing. The polynomial degree $m$ is determined by the security level of the underlying encryption scheme, and $\ell$ is usually determined by application scenarios. As a result, we tend to seek a prime $q$ that satisfies Equation 4 with the given $m$ and $\ell$. More precisely, we should make sure that there exist $\ell$ distinct values $\beta_{k}<q$ such that the multiplicative order of $\beta_{k}$ of the modulo $q$ is $2 \ell$, i.e., $\beta_{k}^{2 \ell}=1 \bmod q$. Empirically, we have found many primes that satisfy this requirement. In Table 3 , we present some examples of practical $m$ and $\ell$ for the matrix product functionality. Specifically, the underlying encryption scheme should provide at least 80 -bit security level when $m=4096$, according to the security analysis from $[14,18]$.

\subsection{Efficient Double Unpacking}

The double unpacking function is defined as $\pi_{\mathrm{w}}^{-1}: \mathbb{R}_{q} \mapsto \mathbb{Z}_{q}^{\ell}$, which is used by the client to extract $\ell$ inner products after decrypting the ciphertexts received from the server. Suppose the polynomial $A=$ $\sum_{i=0}^{m-1} a_{i} X^{i}$ is decrypted from one of the ciphertext that computed by the server. To extract $\ell$ inner products from $A$, mathematically, the client must compute the $(d-1)$-th coefficient of $A \bmod F_{k}$ for each modulo $F_{k}=X^{d}+\beta_{k}$. This can be accomplished by using the unpacking function of the CRT packing $\pi_{\mathrm{crt}}^{-1}$ : take the modulo $A$ $\bmod F_{k}$; then keep only the $(d-1)$-th coefficient of the resulting polynomial and discard the remains. But the effort of computing the discarded coefficients becomes meaningless.

We now present a faster implementation of $\pi_{\mathrm{w}}^{-1}$ by examining the algebraic property $\left(-\beta_{k}\right)^{t-1} X^{d-1}=X^{t d-1} \bmod X^{d}+\beta_{k}$ for positive $t>0$. This gives us a way to compute the $(d-1)$-th coefficient of $A \bmod X^{d}+\beta_{k}$ directly. Specifically, we compute the $(d-1)$-th coefficient of $A \bmod X^{d}+\beta_{k}$ as follows

$$
a_{d-1}+a_{2 d-1}\left(-\beta_{k}\right)+\cdots+a_{\ell d-1}\left(-\beta_{k}\right)^{\ell-1} .
$$

Because $\beta_{k}$ s are known during the key generation, the values $\left(-\beta_{k}\right)^{2}, \cdots,\left(-\beta_{k}\right)^{\ell-1}$ can be computed once and reused for many unpackings. For the case of secure matrix product, thousands of unpackings is usually required, and thus Equation 5 can reduce the computational cost at the client's side significantly. From our empirical results $(\$ 6.3), \pi_{\mathrm{w}}^{-1}$ that uses Equation 5 was about $25-1000$ times faster than using $\pi_{\mathrm{crt}}^{-1}$ directly.

\section{SECURE MATRIX PRODUCT PROTOCOL}

With the optimizations presented in $\S 4$, we now present our secure matrix product protocol SMP in Figure 4. It is noteworthy that we fix the matrix size of $\mathbf{A}$ to $\ell \times d$ and the size of $\mathbf{B}$ to $d \times \ell$ for the sake of simplicity. The protocol in Figure 4 can be easily extended for general matrices, as described later.

The matrix product $\mathrm{AB}$ is computed through inner products between the row vectors of $A$ and the column vectors of $B$. Specifically, the client processes rows of A with $\vec{\pi}_{\mathrm{w}}$ before doing encryption in Step 2. This step produces one ciphertext ctx which is sent to the server. In Step 3, the server applies $\overleftarrow{\pi}_{\mathrm{w}}$ to $\ell$-copies of each column of $\mathbf{B}$, and then multiplies the packed copies to ctx, resulting a ciphertext of one row of the product matrix. In Step 4, uniform random polynomials $\left\{R_{j, k}\right\}_{k \in[\ell]}$ are sampled by the server to prevent extra information from being learned by the client. Also, the server stores the proper coefficients, i.e., $R_{j, k}[d-1]$ for $k \in[\ell]$, into its share $\mathrm{C}^{1}$. In Step 6, the client decrypts all the ciphertexts and uses $\pi_{\mathrm{w}}^{-1}$ to obtain its share $\mathrm{C}^{0}$. We now show that $\mathrm{C}^{0}+\mathrm{C}^{1}=\mathrm{AB} \bmod q$.

TheOREM 5.1. The protocol of Figure 4 computes correctly the functionality $\mathcal{F}_{\text {MP }}$ of Figure 2.

Proof. In Step 2, the client processes the rows of A with $\vec{\pi}_{\mathrm{w}}$. According to Theorem 4.2 , ctx $_{j}$ of Step 3 gives the inner products between the rows of $\mathrm{A}$ and $j$-th column of $\mathrm{B}$, which forms the $j$-th row of $A B$. By iterating all columns of $B$, the matrix product $A B$

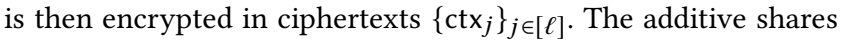


$\mathrm{C}^{0}[j, k]=\mathrm{AB}[j, k]-R_{j, k}[d-1]$ are constructed in Step 4 by homomorphic subtractions, and $R_{j, k}[d-1]$ is kept in $\mathrm{C}^{1}[j, k]$. Thus we have $\mathrm{C}^{0}+\mathrm{C}^{1}=\mathrm{AB} \bmod q$.

THEOREM 5.2. The protocol of Figure 4 privately implements the functionality $\mathcal{F}_{\mathrm{MP}}$ of Figure 2 under the semi-honest setting.

We defer explanation of the security proof to the Appendix A. General Case and Complexity Analysis. For the case of general matrices, $\mathrm{A}$ and $\mathbf{B}$ can be partitioned into block matrices $\left\{\mathrm{A}_{i k}\right\}$ and $\left\{\mathbf{B}_{k j}\right\}$ where the size of $\mathbf{A}_{i k}$ is $\ell \times d$ and the size of $\mathbf{B}_{k j}$ is $d \times \ell$ Zero-padding might be used to align the size. Now, the client must process each block $A_{i k}$ in Step 2. Then AB is computed through a summation of products of the block matrices, i.e., $\sum_{k} \mathbf{A}_{i k} \mathbf{B}_{k j}$. The block-matrix product $\mathbf{A}_{i k} \mathbf{B}_{k j}$ is computed in Step 3 of Figure 2, and the summation can be accomplished from homomorphic additions. Thus, the correctness of Theorem 5.1 follows. Furthermore, no extra interaction between the protocol players is introduced. Therefore, Theorem 5.2 follows, too.

In total, the client processes $O\left(n_{1} n_{2} / m\right)$ blocks. The server operates $O\left(n_{1} n_{2} n_{3} / m\right)$ homomorphic multiplications and additions, resulting $O\left(n_{1} n_{3} / \ell\right)$ ciphertexts which will be transferred to the client. In Table 1 , we summarize and compare the complexity of our method with other homomorphic encryption-based methods. Although the computational complexity of our method are in the same order with the AHE-based method of SecureML [28] our method can provide a considerable acceleration given the fact that $m$ is usually a large value, e.g., $m \geq 2^{12}$. Suppose $n_{1}, n_{2}$, and $n_{3}=128$ and $m=2^{12}$. For our method, the server only operate 512 homomorphic operations as opposed to the $2.0 \times 10^{6}$ homomorphic operations of the AHE-based method.

Special Case: Matrix-Vector Product. When $n_{1}=1$ (i.e., A becomes a single row matrix), the matrix product $\mathrm{AB}$ can be specially regarded as the matrix-vector product. The algorithm of Figure 4 does cover this special case by zero-padding A, although with a small modification, the complexity of the algorithm can be improved in this setting. That is, in Step 2 of Figure 4, the client sends a ciphertext of $\ell$-copies of $\mathrm{A}, \operatorname{ctx}=\operatorname{Enc}\left(\vec{\pi}_{\mathrm{w}}(\mathrm{A}[0,:], \ldots, \mathrm{A}[0,:])\right)$. In Step 3, the server operates the homomorphic multiplication with $\ell$ columns of B instead of just one, i.e.,

$$
\operatorname{ctx}_{0}=\operatorname{ctx} \otimes \pi_{\mathrm{w}}(\mathrm{B}[:, 0], \mathrm{B}[:, 1], \ldots, \mathrm{B}[:, \ell-1]) .
$$

The remaining steps of the algorithm follows. In this case, the server performs $O\left(n_{2} n_{3} / m\right)$ homomorphic operations, and transfers $O\left(n_{3} / \ell\right)$ ciphertexts to the client.

\section{EVALUATIONS}

\subsection{Setups}

Implementations. We implemented SMP using HElib [32]. Our implementation is freely available from https://github.com/OpenSMP/ SMP. The parameters $m=4096$ and $q=70913$ were used to provide $\ell=128$ slots. The other parameters of HElib were set properly to provide at least $\kappa=80$-bit security level. A single ciphertext under this setting was about 64 kilobytes. Additionally, $m=8192$ and $q=84481$, which can provide at least $\kappa=300$-bit security level, were also used in the micro-benchmarks of SMP (Table 4).
Let sk be a private key and evk be the corresponding evaluation key of the underlying homomorphic encryption scheme. The client holds the private key sk.

-Input of client: private matrix $\mathrm{A} \in \mathbb{Z}_{q}^{\ell \times d}$

-Input of server: private matrix $\mathrm{B} \in \mathbb{Z}_{q}^{d \times \ell}$

-Output of client: additively shared matrix $\langle\mathrm{AB}\rangle_{0} \in \mathbb{Z}_{q}^{\ell \times \ell}$

-Output of server: additively shared matrix $\langle\mathrm{AB}\rangle_{1} \in \mathbb{Z}_{q}^{\ell \times \ell}$

1: The client and the server set $\mathrm{C}^{0}, \mathrm{C}^{1} \in \mathbb{Z}_{q}^{\ell \times \ell}$ to a zero matrix, respectively.

2: The client processes rows of $\mathrm{A}$ and sends the ciphertext ctx $=$ $\operatorname{Enc}\left(\vec{\pi}_{\mathrm{w}}(\mathrm{A}[0,:], \ldots, \mathrm{A}[\ell-1,:])\right)$ to the server, attached with its evaluation key evk.

3: For each column $\mathrm{B}[:, j]$, the server computes

$$
\operatorname{ctx}_{j}=\operatorname{ctx} \otimes \overleftarrow{\pi}_{\mathrm{w}}(\underbrace{\mathrm{B}[:, j], \mathrm{B}[:, j], \ldots, \mathrm{B}[:, j]}_{\ell \text {-copies }})
$$

4: For each $\operatorname{ctx}_{j}$, the server randomizes it by

$$
\operatorname{ctx}_{j}^{\prime}=\operatorname{ctx}_{j} \ominus \pi_{\mathrm{crt}}\left(R_{j, 0}, \ldots, R_{j, \ell-1}\right),
$$

where $R_{j, k} \stackrel{\$}{\leftarrow} \mathbb{Z}_{q}[X] /\left(F_{k}\right)$. Also, the server stores the $(d-1)$-th coefficient in the $j$-th row of $\mathrm{C}^{1}[j, k]=R_{j, k}[d-1]$ for $k \in[\ell]$.

5: The server sends back $\ell$ ciphertexts $\left\{\text { ctx }_{j}^{\prime}\right\}_{j \in[\ell]}$ to the client.

6: For each $\mathrm{ctx}_{j}^{\prime}$, the client decrypts it and uses $\pi_{\mathrm{w}}^{-1}$ presented in $\S 4.4$ to extract $\ell$ values, which are placed in the $j$-th row of $\mathrm{C}^{0}$. 7: The client and the server output $\mathrm{C}^{0}$ and $\mathrm{C}^{1}$, respectively.

\section{Figure 4: Secure matrix product protocol SMP.}

We compared SMP with three existing methods, including the OT-based method, the AHE-based method from SecureML and the SwHE-based method from MiniONN. Specifically, we used the EMP-toolkit [37] to implement the OT-based method of SecureML, using 16-bit inputs for a fair comparison because $\log _{2} q \approx 16$. For the AHE-based method, we instantiated the AHE with the DGK scheme [7] with a 1024-bit RSA modulus using the implementation from [9]. For the SwHE-based method of MiniONN, we used the HElib and parameters were set as $m=4096$ and $q=65537$, aiming to provide $\ell=4096$ plaintext slots.

Computation and Communication Environments. We conducted extensive experiments using a various types of computing machines and under two network settings.

- Server specifications. We used one AWS c5.18xlarge instance of 72 virtual CPUs (vCPUs) @3.00 GHz and 25 Gbps outgoing bandwidth as the server node.

- Client specifications. The concurrent clients' requests were equally launched from five AWS c4.8xlarge instances of 36 vCPUs @2.90 GHz for each instance. Also, we ran experiments on a weak device, i.e., Raspberry Pi model B v1.2 of one CPU @900 MHz.

- LAN setting. For the LAN setting, all the AWS instances were launched inside the same region. The ping delay was about $2.5 \mathrm{~ms}$.

- WAN setting. For the WAN setting, the server instance was launched in west US and the client instances were launched 
Table 4: Micro-benchmarks of SMP using one single access. The performance numbers were averaged from 50 runs. $m$ and $\ell$ are parameters of the underlying encryption scheme, and $\kappa$ denotes the security level.

\begin{tabular}{|c|c|c|c|c|c|c|c|c|c|c|}
\hline \multirow{2}{*}{$(m, \ell, \kappa)$} & \multirow{2}{*}{$n_{1} \times n_{3}$} & \multirow{2}{*}{$\vec{\pi}_{\mathrm{w}}$} & \multirow{2}{*}{ ENC } & \multirow{2}{*}{ EVA } & \multirow{2}{*}{$\mathrm{DEC}$} & \multirow{2}{*}{$\pi_{\mathrm{w}}^{-1}$} & \multicolumn{2}{|c|}{ Total Time } & \multicolumn{2}{|c|}{ Communication } \\
\hline & & & & & & & LAN & WAN & sent by client & sent by server \\
\hline \multirow{3}{*}{$(4096,128,80)$} & $128^{2}$ & $27.9 \mathrm{~ms}$ & $16.1 \mathrm{~ms}$ & $205 \mathrm{~ms}$ & $233 \mathrm{~ms}$ & $28.9 \mathrm{~ms}$ & $2.45 \mathrm{sec}$ & $3.30 \mathrm{sec}$ & $0.25 \mathrm{MB}$ & $8 \mathrm{MB}$ \\
\hline & $256^{2}$ & $43.8 \mathrm{~ms}$ & $26.2 \mathrm{~ms}$ & $670 \mathrm{~ms}$ & $744 \mathrm{~ms}$ & $83.8 \mathrm{~ms}$ & $7.25 \mathrm{sec}$ & $8.22 \mathrm{sec}$ & $0.5 \mathrm{MB}$ & $32 \mathrm{MB}$ \\
\hline & $512^{2}$ & $81.1 \mathrm{~ms}$ & $44.9 \mathrm{~ms}$ & $2.63 \mathrm{sec}$ & $2.82 \mathrm{sec}$ & $299 \mathrm{~ms}$ & $26.4 \mathrm{sec}$ & $27.7 \mathrm{sec}$ & $1.0 \mathrm{MB}$ & $128 \mathrm{MB}$ \\
\hline \multirow{3}{*}{$(8192,256,300)$} & $256^{2}$ & $67.9 \mathrm{~ms}$ & $31.8 \mathrm{~ms}$ & $614 \mathrm{~ms}$ & $770 \mathrm{~ms}$ & $197 \mathrm{~ms}$ & $9.37 \mathrm{sec}$ & $10.7 \mathrm{sec}$ & $0.5 \mathrm{MB}$ & $32 \mathrm{MB}$ \\
\hline & $512^{2}$ & $108 \mathrm{~ms}$ & $48.4 \mathrm{~ms}$ & $2.46 \mathrm{sec}$ & $2.88 \mathrm{sec}$ & $720 \mathrm{~ms}$ & $29.1 \mathrm{sec}$ & $30.4 \mathrm{sec}$ & $1.0 \mathrm{MB}$ & $128 \mathrm{MB}$ \\
\hline & $1024^{2}$ & $140 \mathrm{~ms}$ & $75.9 \mathrm{~ms}$ & $9.80 \mathrm{sec}$ & $11.0 \mathrm{sec}$ & $2.81 \mathrm{sec}$ & $65.4 \mathrm{sec}$ & $110 \mathrm{sec}$ & $2.0 \mathrm{MB}$ & $512 \mathrm{MB}$ \\
\hline
\end{tabular}

Table 5: Speedup of the unpacking $\pi_{\mathrm{w}}^{-1}$ due to the precomputation from Equation 5. The SwHE parameters $m$ and $q$ follow Table 3.

\begin{tabular}{c|cc|cc}
\hline \multirow{2}{*}{ \#slots $\ell$} & \multicolumn{2}{|c}{ c4.x8large } & \multicolumn{2}{c}{ Raspberry Pi } \\
\cline { 2 - 5 } & $\begin{array}{c}\text { with } \\
\text { pre-comp. }\end{array}$ & $\begin{array}{c}\text { w/o-comp. } \\
\text { pre-com }\end{array}$ & $\begin{array}{c}\text { with } \\
\text { pre-comp. }\end{array}$ & $\begin{array}{c}\text { w/o } \\
\text { pre-comp. }\end{array}$ \\
\hline 16 & $0.008 \mathrm{~ms}$ & $31.1 \mathrm{~ms}$ & $0.360 \mathrm{~ms}$ & $549 \mathrm{~ms}$ \\
64 & $0.032 \mathrm{~ms}$ & $33.2 \mathrm{~ms}$ & $1.33 \mathrm{~ms}$ & $573 \mathrm{~ms}$ \\
128 & $0.120 \mathrm{~ms}$ & $33.3 \mathrm{~ms}$ & $5.03 \mathrm{~ms}$ & $612 \mathrm{~ms}$ \\
256 & $0.437 \mathrm{~ms}$ & $36.3 \mathrm{~ms}$ & $21.9 \mathrm{~ms}$ & $694 \mathrm{~ms}$ \\
512 & $1.68 \mathrm{~ms}$ & $41.6 \mathrm{~ms}$ & $99.9 \mathrm{~ms}$ & $853 \mathrm{~ms}$ \\
\hline
\end{tabular}

in east Asia. The bandwidth was about $114 \mathrm{Mbps}$ with about 110 ms ping delay.

Measurements. We measured the end-to-end running time of each MT generation method using a high resolution clock (i.e., the standard chrono library). For the OT-based method, the time for computing the OT-extension [2] is included. For SMP, the computing time of Equation 5 is included while the key generation time is excluded. We also measured the total amount of data transferred by each method. Moreover, we provide five more micro-benchmarks of SMP. That is, the computation time of packing $\vec{\pi}_{\mathrm{w}}$, encryption (ENC), decryption (DEC), and unpacking $\pi_{\mathrm{w}}^{-1}$ on the client's side, and the evaluation (EVA) time on the server's side.

Concurrent Accesses. To mimic highly concurrent accesses, we used a shell script to launch the MT generation requests equally from five AWS instances. For instance, to mimic the 1000 concurrent accesses, we launched 200 requests from each instances. Recall that each c4.x8large instance possesses only 36 vCPUs. This means the CPU resources for each request at the client's side might be smaller than $2.90 \mathrm{GHz}$ when we launched more than 36 requests on these instances. This CPUs sharing should contribute a part of the performance decreases in our experiments.

\subsection{Micro-benchmarks}

The micro-benchmarks of SMP is given in Table 4. Additionally, we used a higher security level $\kappa=160$ in this experiment to demonstrate its performance growth with respect to $\kappa$. By the virtue of our new packing method and its extension, the computation on the server' side (i.e., the EVA column) was very fast.

\subsection{Faster Unpacking Optimization}

We can instantiate the double unpacking function $\pi_{\mathrm{w}}^{-1}$ using the unpacking function $\pi_{\text {crt }}^{-1}$, but this will introduce an expensive computation at the client's side. In $\S 4$.4, we present to use a pre-computed table to eliminate the needs of $\pi_{\mathrm{crt}}^{-1}$. Experiment results (Table 5) show that this optimization can significantly reduce the computation burden of the client. This experiment was conducted on two devices, the powerful AWS instance and the much weaker Raspberry Pi. We can see that the unpacking $\pi_{\mathrm{w}}^{-1}$ from using Equation 5 was much faster than using $\pi_{\mathrm{crt}}^{-1}$.

\subsection{Comparison to Other MT Generation Methods}

The comparison results to the existing MT generation methods are given in Table 6. From Table 6, we know that SMP outperformed these methods in terms of MT generation time, especially when many clients access to the server concurrently.

For the AHE-based method, the server needs to operate $n_{1} n_{2} n_{3}$ homomorphic operations while our method requires only $n_{1} n_{2} n_{3} / m$ homomorphic operations. Since the parameter $m$ of the underlying encryption scheme is usually large, e.g., $m \geq 2^{12}$, our method can provide a considerable boost, e.g., it was about $8-12$ times faster than the AHE-based method according to our benchmarks. Although the consumed network traffic of SMP was $2-3$ times larger than that of the AHE-based method, the absolute amount of the network traffic was still small enough to be transferred through a narrow bandwidth within a reasonable time.

Comparing to the OT-based method, SMP only exchanged about $1.9 \%$ of data of the OT-based method. As a result, when many clients access to the server concurrently, the running time of SMP was much faster than the OT-based method, e.g., about $66-97$ times faster when the number of concurrent accesses was 10. Moreover, the performance of our method was less sensitive to the network latency than the OT-based method. That is because our method is a single-round protocol while the OT-based method requires multiple rounds of communication between the client and the server. 
Table 6: Comparing the performances of SMP with the AHE-based method and the OT-based method from SecureML [28], and the SwHE-based method from MiniONN [23], under LAN and WAN, respectively.

\begin{tabular}{|c|c|c|c|c|c|}
\hline \multirow{2}{*}{$\begin{array}{c}\text { Dimension } \\
n_{1}, n_{2}, n_{3} \\
\end{array}$} & \multirow{2}{*}{ Method } & \multicolumn{3}{|c|}{ MT Generation Time (LAN/WAN) } & \multirow{2}{*}{ Communication } \\
\hline & & 1 client & 10 clients & 50 clients & \\
\hline \multirow{4}{*}{$128,128,128$} & AHE & $26.3 \mathrm{sec} / 26.9 \mathrm{sec}$ & $26.1 \mathrm{sec} / 26.7 \mathrm{sec}$ & $33.2 \mathrm{sec} / 33.0 \mathrm{sec}$ & $3.98 \mathrm{MB}$ \\
\hline & OT & $4.07 \mathrm{sec} / 60.8 \mathrm{sec}$ & $140 \mathrm{sec} / 168 \mathrm{sec}$ & $389 \mathrm{sec} / 485 \mathrm{sec}$ & $432 \mathrm{MB}$ \\
\hline & SwHE & $123 \mathrm{sec} / 124 \mathrm{sec}$ & $123 \mathrm{sec} / 127 \mathrm{sec}$ & $146 \mathrm{sec} / 151 \mathrm{sec}$ & $32.3 \mathrm{MB}$ \\
\hline & Ours & $2.49 \mathrm{sec} / 3.26 \mathrm{sec}$ & $2.54 \mathrm{sec} / 3.35 \mathrm{sec}$ & $3.53 \mathrm{sec} / 3.36 \mathrm{sec}$ & $8.25 \mathrm{MB}$ \\
\hline \multirow{4}{*}{$256,128,256$} & AHE & $95.1 \mathrm{sec} / 95.3 \mathrm{sec}$ & $94.6 \mathrm{sec} / 95.0 \mathrm{sec}$ & $122 \mathrm{sec} / 123 \mathrm{sec}$ & $11.9 \mathrm{MB}$ \\
\hline & OT & $16.1 \mathrm{sec} / 235 \mathrm{sec}$ & $588 \mathrm{sec} / 656 \mathrm{sec}$ & $0.426 \mathrm{hr} / 0.472 \mathrm{hr}$ & $1664 \mathrm{MB}$ \\
\hline & SwHE & $486 \mathrm{sec} / 489 \mathrm{sec}$ & $495 \mathrm{sec} / 498 \mathrm{sec}$ & $545 \mathrm{sec} / 545 \mathrm{sec}$ & $129 \mathrm{MB}$ \\
\hline & Ours & $7.38 \mathrm{sec} / 8.21 \mathrm{sec}$ & $7.41 \mathrm{sec} / 8.63 \mathrm{sec}$ & $10.8 \mathrm{sec} / 11.0 \mathrm{sec}$ & $32.5 \mathrm{MB}$ \\
\hline \multirow{4}{*}{$512,128,512$} & AHE & $361 \mathrm{sec} / 361 \mathrm{sec}$ & $356 \mathrm{sec} / 359 \mathrm{sec}$ & $507 \mathrm{sec} / 510 \mathrm{sec}$ & $39.8 \mathrm{MB}$ \\
\hline & OT & $57.5 \mathrm{sec} / 917 \mathrm{sec}$ & $0.723 \mathrm{hr} / 0.732 \mathrm{hr}$ & $1.59 \mathrm{hr} / 1.82 \mathrm{hr}$ & $6520 \mathrm{MB}$ \\
\hline & SwHE & $0.522 \mathrm{hr} / 0.538 \mathrm{hr}$ & $0.556 \mathrm{hr} / 0.574 \mathrm{hr}$ & $0.560 \mathrm{hr} / 0.570 \mathrm{hr}$ & $513 \mathrm{MB}$ \\
\hline & Ours & $26.6 \mathrm{sec} / 27.5 \mathrm{sec}$ & $26.8 \mathrm{sec} / 29.1 \mathrm{sec}$ & $41.2 \mathrm{sec} / 41.4 \mathrm{sec}$ & $129 \mathrm{MB}$ \\
\hline
\end{tabular}
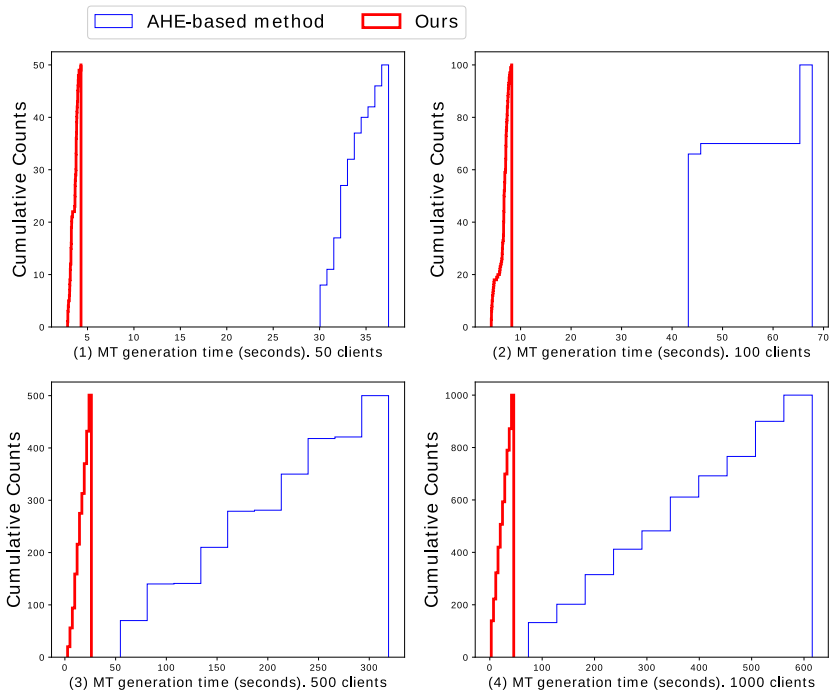

Figure 5: Cumulative histograms of the performances of SMP and the AHE-based method of [28] under 50, 100, 500, and 1000 concurrent accesses. The size of matrices was $128 \times$ 128.

SMP was about 38 - 70 times faster than, and consumed only one-fourth network traffic of the method of MiniONN. The reason for this speedup is two-fold. That is, less unpackings are needed by SMP, that is a quadratic number of unpackings $n_{1} n_{3} / \ell$ as opposed to the cubic number of unpackings $n_{1} n_{2} n_{3} / \ell$ of their method. Moreover, our faster unpacking optimization (i.e., Equation 5) even accelerates the computation on the client's side.

\subsection{Handling 1000 Concurrent Accesses}

To show the feasibility of using the proposed method under the high concurrency setting, we benchmarked SMP with more concurrent accesses under the LAN setting. Specifically, for each of the five AWS c4.8xlarge instances, we launched 10, 20, 100, and $200 \mathrm{MT}$ generation requests to the server instance, and recorded the total running time for each request. In the other words, the server instance handled concurrent accesses of 50,100, 500 and 1000 using its 72 vCPUs. We only compare with the AHE-based method of [28] because of the OT-based method and the SwHE-based method would take too much time under the high concurrency setting. The results are shown in Figure 5. The server handled 100 concurrent accesses within 10 seconds (i.e., the 2-rd plot of Figure 5). Therefore, if we want to handle 1000 concurrent accesses within 10 seconds, we need about 10 server nodes.

\subsection{Experiments on Constrained Devices}

We also ran the MT generation methods on the Raspberry Pi. However we failed to compile the OT library [37] on the Raspberry Pi. Therefore, only the homomorphic encryption-based methods were considered in this experiment. Matrices of size $128 \times 128$ were used. The total running times are as follows.

- Ours: 16.2 seconds.

- AHE-based method of [28]: 389 seconds.

- SwHE-based method of [23]: more than 23 minutes.

We can see that our method can perform better than the existing HE-based methods on resource constrained devices.

\section{APPLICATIONS TO MACHINE LEARNING}

In this section, we demonstrate how the existing privacy-preserving machine learning frameworks can benefit from SMP under the high concurrency setting. Notice that these two frameworks were originally designed for the one-client setting, and thus no empirical benchmarks of these frameworks under the concurrent setting are reported. As a result, we estimate their performances under 
Table 7: Using SMP to improve the pre-computation stage of SecureML. The mini-batch size $B$ was fixed as 128 as SecureML. The performance numbers of the methods of SecureML are taken from their paper [28].

\begin{tabular}{cc|cc}
\hline$N, D, t$ & Method & Time (LAN/WAN) & Commu. \\
\hline \multirow{3}{*}{$10^{4}, 100,156$} & AHE & $248.4 \mathrm{sec} / 252.9 \mathrm{sec}$ & $20 \mathrm{MB}$ \\
& OT & $7.9 \mathrm{sec} / 420.2 \mathrm{sec}$ & $1.9 \mathrm{~GB}$ \\
\cline { 2 - 4 } & Ours & $23.4 \mathrm{sec} / 30.2 \mathrm{sec}$ & $159 \mathrm{MB}$ \\
\hline \hline \multirow{3}{*}{$10^{5}, 100,1563$} & AHE & $2437.1 \mathrm{sec} / 2478.1 \mathrm{sec}$ & $200 \mathrm{MB}$ \\
& OT & $88.0 \mathrm{sec} / 4125.1 \mathrm{sec}$ & $19 \mathrm{~GB}$ \\
\cline { 2 - 4 } & Ours & $168 \mathrm{sec} / 187 \mathrm{sec}$ & $785 \mathrm{MB}$ \\
\hline
\end{tabular}

the concurrent setting using the empirical results in Table 6, and compared the estimated values with the empirical results of SMP.

\subsection{Application to Private Machine Learning Model Training}

SecureML is a secure computation framework that is originally designed for training machine learning models from a dataset that is already shared additively between two collusion-free servers. The private model training is performed between the two collusion-free servers using OT-based secure matrix multiplication protocol, and other cryptographic tools such as Yao's garbled circuit [37, 39].

Specifically, SecureML uses the mini-batch stochastic gradient descent (SGD) of a batch size $B>0$ to train their models from a dataset $\mathrm{X} \in \mathbb{Z}^{N \times D}$ within $t$ steps. Here, $N$ indicates the number of data points in the dataset and $D$ indicates the number of features. As suggested by SecureML, when using SGD for some classes of machine learning algorithms, e.g., linear regression and logistic regression, the SGD computation involves a matrix product UV, where the size of $\mathbf{U}$ is $B \times D$ and the size of $\mathbf{V}$ is $D \times t$. The value of $B$ is usually a few hundreds, e.g., $B=128$ as in SecureML, and the value of $t$ is usually set such that $B t>N$.

We now show that SMP can be a better alternative for performing secure matrix multiplication in SecureML, especially under the high concurrency setting. Specifically, we compared the computation time and communication cost with the performance numbers presented in their paper [28]. Notice that, 64-bit inputs were used in SecureML while the plaintext precision of our encryption scheme was $\log _{2} q \approx 16$. To have a fair comparison, we use the techniques $[15,24]$ to achieve the same level of 64-bits precision. The comparison details are given in Table 7. It is apparent that our method is more efficient, especially when the matrix size is large and the number of concurrent accesses is more than one. Specifically, our method was about 3-21 times faster than the AHE-based method when the matrix size was $100 \times 1563$. Also, it was $5-35$ times faster than the OT-based method and consumed only about $4.0 \%-5.3 \%$ of the OT-based method.

\subsection{Application to Private Deep Neural Networks Evaluation}

MiniONN is a two-stage secure computation framework allows evaluating a trained neural network privately. The evaluation of
Table 8: Using SMP to improve the pre-computation stage of MiniONN. The performance numbers of the method of MiniONN are taken from their paper [23].

(a) The matrix products involved in NN-CIFAR.

\begin{tabular}{c|cccc}
\hline Layer & 1 & 3 & 6 & 8 \\
\hline A & $1024 \times 27$ & $1024 \times 576$ & $256 \times 576$ & $256 \times 576$ \\
B & $27 \times 64$ & $576 \times 64$ & $576 \times 64$ & $576 \times 64$ \\
\hline \hline Layer & 11 & 13 & 15 & 17 \\
\hline A & $64 \times 576$ & $64 \times 64$ & $64 \times 64$ & $1 \times 1024$ \\
B & $576 \times 64$ & $64 \times 64$ & $64 \times 16$ & $1024 \times 10$ \\
\hline
\end{tabular}

(b) Performances under a single access.

\begin{tabular}{ccc}
\hline Method & Time (LAN/WAN) & Commu. \\
\hline MiniONN & $472 \mathrm{sec} /-$ & $3046 \mathrm{MB}$ \\
\hline Ours & $24.5 \mathrm{sec} / 26.6 \mathrm{sec}$ & $137 \mathrm{MB}$ \\
\hline
\end{tabular}

a neural network usually requires to compute products of largescale matrices. During the pre-computation stage, MiniONN uses a SwHE scheme and the CRT-packing to perform secure matrix multiplication.

We experimentally show that SMP is a more practical option for MiniONN to perform secure matrix multiplication. Specifically, we take the 17-layer neural network from MiniONN as an example. This network was originally designed for classifying the CIFAR-10 dataset [21], and thus we designate it as NN-CIFAR. In Table 8a, we list up all the matrix products involved in NN-CIFAR where the matrix $A$ is the private input from the client and $B$ is the private input from the service operator. We compared the computation time and communication cost of our protocol with MiniONN. The comparison details are shown in Table 8b. It is apparent that SMP considerably reduced the computation time and the communication cost of the pre-computation stage of MiniONN for evaluating NNCIFAR, i.e., saving more than $95 \%$ of the computation time and communication cost.

\section{CONCLUSION}

In this paper, we presented SMP for efficiently generating multiplication triples for multiplying two matrices. SMP is built from ring-based homomorphic encryption with three algorithmic and implementation optimizations. Our optimizations significantly reduce the computation time, both at the severs side and at the client's side. Moreover, the communication cost of our method is considerably less than the existing OT-based methods. According to our experimental results, SMP outperformed the existing methods under the high concurrency setting. We also applied SMP to two frameworks of privacy-preserving machine learning, i.e., SecureML and MiniONN. The experimental results showed that SMP can reduce MT generation time of these frameworks by more than $74 \%-97 \%$. In concluding, we consider that SMP can help forwarding the deployment of more practical and usable secure two-party computation to machine learning-based online applications. 


\section{ACKNOWLEDGMENTS}

This work was supported by JST CREST program "Advanced Core Technologies for Big Data Integration”. Wen-jie Lu is supported in Grant-in-Aid for JSPS Fellows from Japan Society for the Promotion of Science.

\section{A PROOF OF THEOREM 5.2}

We now prove Theorem 5.2, that is, SMP privately implements the matrix product functionality $\mathcal{F}_{M P}$ described in Figure 2 under the semi-honest assumption.

Proof. Security Against a Semi-Honest Server. We first prove security against a semi-honest server. Given the fact that, the server's view during the protocol execution consists only of ciphertexts, and thus the security against a semi-honest server can be reduced to the semantic security of the underlying encryption scheme. We write $\mathcal{A}^{1}$ to denote the adversarial server, and construct an ideal-world simulator $\mathcal{S}^{0}$ as follows:

(1) The simulator $\mathcal{S}^{0}$ receives the matrix $\mathrm{A}$ from the environment $\mathcal{E}$ and sends $\mathrm{A}$ to TTP for the result $\langle\mathrm{AB}\rangle_{0}$.

(2) The simulator $\mathcal{S}^{0}$ starts running $\mathcal{A}^{1}$ on input A. Then, $\mathcal{S}^{0}$ generates a key-pair $\left(\mathrm{sk}^{\prime}, \mathrm{evk^{ \prime }}\right)$ for the underlying encryption scheme used in the protocol. Next, $\mathcal{S}^{0}$ sends a fresh encryption

$$
\mathrm{ctx}=\operatorname{Enc}\left(\vec{\pi}_{\mathrm{w}}(0, \ldots, 0) ; \mathrm{sk}^{\prime}\right)
$$

of zero vectors to $\mathcal{A}^{1}$, along with evk'.

(3) After $\mathcal{A}^{1}$ replies with the result, $\mathcal{S}^{0}$ outputs $\langle\mathrm{AB}\rangle_{0}$. The view of the server during the real execution is $\mathcal{V}^{1}=\{\operatorname{ctx}\}$ while its view in the ideal world is $\hat{V}^{1}=\{\mathrm{ct \hat {x }}\}$. By semantic security of the underlying encryption scheme, $\mathcal{V}^{1}$ is computationally indistinguishable from $\hat{V}^{1}$.

Security Against a Semi-Honest Client. Next, we prove security against a semi-honest client. Similarly, we write $\mathcal{A}^{0}$ to denote the adversarial client and construct an ideal-world simulator $\mathcal{S}^{1}$ that works as follow:

(1) The simulator $\mathcal{S}^{1}$ receives the matrix B from the environment $\mathcal{E}$, and sends $\mathrm{B}$ to TTP for the result $\langle\mathrm{AB}\rangle_{1}$.

(2) The simulator $\mathcal{S}^{1}$ starts running $\mathcal{A}^{0}$ on input $\mathrm{B}$, and receives ctx along with the evaluation key evk.

(3) For each $j \in[\ell], \mathcal{S}^{1}$ computes a ciphertext $\hat{c t x}_{j}^{\prime}=\operatorname{ctx} \oplus \hat{R}_{j}$ where polynomial $\hat{R}_{j} \stackrel{\$}{\leftarrow} \mathbb{Z}_{q}[X] /\left(X^{m}+1\right)$.

(4) The simulator $\mathcal{S}^{1}$ sends the ciphertexts $\left\{\mathrm{ctxx}_{j}^{\prime}\right\}_{j \in[\ell]}$ to $\mathcal{A}^{0}$, and then outputs $\langle\mathrm{AB}\rangle_{1}$.

The view of the client during the real execution consists of $\ell$ independent degree- $m$ polynomials with coefficients distributed uniformly over $\mathbb{Z}_{q}$. That is exactly how $\mathcal{S}^{1}$ creates client's view in the ideal-world. Thus, the client's view in the real execution and its view in the ideal-world are identically distributed.

\section{REFERENCES}

[1] Benny Applebaum, Yuval Ishai, and Eyal Kushilevitz. October 22-25, 2011. How to Garble Arithmetic Circuits. In IEEE 52nd FOCS 2011. Palm Springs, California, USA, $120-129$.

[2] Gilad Asharov, Yehuda Lindell, Thomas Schneider, and Michael Zohner. November 4-8, 2013. More efficient oblivious transfer and extensions for faster secure computation. In 2013 ACM SIGSAC CCS'13. Berlin, Germany, 535-548.
[3] Donald Beaver. August 27-31, $1995 . \quad$ Precomputing Oblivious Transfer. In CRYPTO'95, Proceedings. Santa Barbara, California, USA, 97-109.

[4] Zvika Brakerski, Craig Gentry, and Vinod Vaikuntanathan. January 8-10, 2012. (Leveled) fully homomorphic encryption without bootstrapping. In Innovations in Theoretical Computer Science 2012. Cambridge, Massachusetts, USA, 309-325.

[5] Ran Canetti. 2000. Security and Composition of Multiparty Cryptographic Protocols. f. Cryptology 13, 1 (2000), 143-202.

[6] Hao Chen, Kim Laine, and Rachel Player. 2017. Simple Encrypted Arithmetic Library - SEAL v2.1. IACR Cryptology ePrint Archive 2017 (2017), 224.

[7] Ivan Damgård, Martin Geisler, and Mikkel Krøigaard. 2009. A correction to 'efficient and secure comparison for on-line auctions'. International fournal of Advancements in Computing Technology 1, 4 (2009), 323-324.

[8] Ivan Damgård, Valerio Pastro, Nigel P. Smart, and Sarah Zakarias. August 19-23, 2012. Multiparty Computation from Somewhat Homomorphic Encryption. In CRYPTO 2012, Proceedings. Santa Barbara, California, USA, 643-662.

[9] Daniel Demmler, Thomas Schneider, and Michael Zohner. February 8-11, 2015. ABY - A Framework for Efficient Mixed-Protocol Secure Two-Party Computation. In 22nd NDSS, 2015. San Diego, California, USA.

[10] Dung Hoang Duong, Pradeep Kumar Mishra, and Masaya Yasuda. 2016. Efficient secure matrix multiplication over LWE-based homomorphic encryption. Tatra Mountains Mathematical Publications 67, 1 (2016), 69-83.

[11] Zekeriya Erkin, Martin Franz, Jorge Guajardo, Stefan Katzenbeisser, Inald Lagendijk, and Tomas Toft. August 5-7, 2009. Privacy-Preserving Face Recognition. In PoPETs 2009, Proceedings. Seattle, WA, USA, 235-253.

[12] Junfeng Fan and Frederik Vercauteren. 2012. Somewhat Practical Fully Homomorphic Encryption. IACR Cryptology ePrint Archive 2012 (2012), 144

[13] Adrià Gascón, Phillipp Schoppmann, Borja Balle, Mariana Raykova, Jack Doerner, Samee Zahur, and David Evans. 2017. Privacy-Preserving Distributed Linear Regression on High-Dimensional Data. PoPETs 2017, 4 (2017), 345-364.

[14] Craig Gentry, Shai Halevi, and Nigel P. Smart. August 19-23, 2012. Homomorphic Evaluation of the AES Circuit. In CRYPTO 2012, Proceedings. Santa Barbara, California, USA, 850-867.

[15] Ran Gilad-Bachrach, Nathan Dowlin, Kim Laine, Kristin E. Lauter, Michael Naehrig, and John Wernsing. June 19-24, 2016. CryptoNets: Applying Neural Networks to Encrypted Data with High Throughput and Accuracy. In ICML 2016, Proceedings. Network, USA, 201-210.

[16] Oded Goldreich. 2009. Foundations of cryptography: volume 2, basic applications. Cambridge University Press.

[17] Shafi Goldwasser and Silvio Micali. May 5-7, 1982. Probabilistic Encryption and How to Play Mental Poker Keeping Secret All Partial Information. In STOC 1982. San Francisco, California, USA, 365-377.

[18] Shai Halevi and Victor Shoup. August 17-21, 2014. Algorithms in HElib. In Advances in Cryptology - CRYPTO 2014 - 34th Annual Cryptology Conference, Proceedings, Part I. Santa Barbara, California, USA, 554-571.

[19] Yan Huang, David Evans, Jonathan Katz, and Lior Malka. August 8-12, 2011. Faster Secure Two-Party Computation Using Garbled Circuits.. In 20th USENIX Security Symposium. San Francisco, California, USA.

[20] Vladimir Kolesnikov and Thomas Schneider. July 7-11, 2008. Improved Garbled Circuit: Free XOR Gates and Applications. In Automata, Languages and Programming, 35th International Colloquium, ICALP 2008, Proceedings, Part II. Reykjavik, Iceland, 486-498.

[21] Alex Krizhevsky and Geoffrey Hinton. 2009. Learning multiple layers of features from tiny images. http://www.cs.utoronto.ca/ kriz/learning-features-2009-TR. pdf.

[22] Chang Liu, Xiao Shaun Wang, Kartik Nayak, Yan Huang, and Elaine Shi. May 17-21, 2015. ObliVM: A programming framework for secure computation. In IEEE SP 2015. San Jose, California, USA, 359-376.

[23] Jian Liu, Mika Juuti, Yao Lu, and N. Asokan. October 30 - November 03, 2017. Oblivious Neural Network Predictions via MiniONN Transformations. In CCS 2017, Proceedings. Dallas, Texas, USA, 619-631.

[24] Wen-jie Lu, Shohei Kawasaki, and Jun Sakuma. February 26-March 1, 2017. Using Fully Homomorphic Encryption for Statistical Analysis of Categorical, Ordinal and Numerical Data. In NDSS 2017. San Diego, California, USA.

[25] Wen-Jie Lu, Yoshiji Yamada, and Jun Sakuma. 2015. Privacy-preserving genomewide association studies on cloud environment using fully homomorphic encryption. BMC medical informatics and decision making 15, 5 (2015), S1.

[26] Vadim Lyubashevsky, Chris Peikert, and Oded Regev. May 30 - June 3, 2010. On ideal lattices and learning with errors over rings. In EUROCRYPT 2010. French Riviera, 1-23.

[27] Pradeep Kumar Mishra, Dung Hoang Duong, and Masaya Yasuda. December 13-15, 2017. Enhancement for Secure Multiple Matrix Multiplications over RingLWE Homomorphic Encryption. In ISPEC 2017, Proceedings. Melbourne, VIC, Australia, 320-330.

[28] Payman Mohassel and Yupeng Zhang. May 22-26, 2017. SecureML: A System for Scalable Privacy-Preserving Machine Learning. In 2017 IEEE Symposium on Security and Privacy, SP 2017. San Jose, California, USA, 19-38. 
[29] Michael Naehrig, Kristin E. Lauter, and Vinod Vaikuntanathan. October 21, 2011 Can homomorphic encryption be practical?. In ACM CCSW 2011, Proceedings. Chicago, Illinois, USA, 113-124.

[30] Valeria Nikolaenko, Stratis Ioannidis, Udi Weinsberg, Marc Joye, Nina Taft, and Dan Boneh. November 4-8, 2013. Privacy-preserving matrix factorization. In CCS 13. Berlin, Germany, 801-812.

[31] M. Sadegh Riazi, Christian Weinert, Oleksandr Tkachenko, Ebrahim M. Songhori, Thomas Schneider, and Farinaz Koushanfar. 2018. Chameleon: A Hybrid Secure Computation Framework for Machine Learning Applications. CoRR abs/1801.03239 (2018). arXiv:1801.03239

[32] Victor Shoup Shai Halevi. 2017. HELib. http://shaih.github.io/HElib. Accessed: 2017-04-10.

[33] Anima Singh and John V. Guttag. August 30 - Sept. 3, 2011. A comparison of non-symmetric entropy-based classification trees and support vector machine for cardiovascular risk stratification. In EMBC 2011. Boston, MA, USA, 79-82.

[34] Nigel P Smart and Frederik Vercauteren. 2014. Fully homomorphic SIMD operations. Designs, codes and cryptography 71, 1 (2014), 57-81.

[35] Ebrahim M Songhori, Siam U Hussain, Ahmad-Reza Sadeghi, Thomas Schneider, and Farinaz Koushanfar. May 17-21, 2015. Tinygarble: Highly compressed and scalable sequential garbled circuits. In 2015 IEEE Symposium on Security and Privacy, SP 2015. San Jose, California, USA, 411-428.

[36] Shuang Wang, Yuchen Zhang, Wenrui Dai, Kristin E. Lauter, Miran Kim, Yuzhe Tang, Hongkai Xiong, and Xiaoqian Jiang. 2016. HEALER: homomorphic computation of ExAct Logistic rEgRession for secure rare disease variants analysis in GWAS. Bioinformatics 32, 2 (2016), 211-218.

[37] Xiao Wang, Alex J. Malozemoff, and Jonathan Katz. 2016. EMP-toolkit: Efficient MultiParty computation toolkit. https://github.com/emp-toolkit.

[38] David J. Wu, Joe Zimmerman, Jérémy Planul, and John C. Mitchell. [n. d.]. PrivacyPreserving Shortest Path Computation. In NDSS.

[39] Andrew C Yao. 3-5 November 1982. Protocols for secure computations. In FOCS 1982. Chicago, Illinois, USA, 160-164.

[40] Masaya Yasuda, Takeshi Shimoyama, Jun Kogure, Kazuhiro Yokoyama, and Takeshi Koshiba. November 4, 2013. Secure pattern matching using somewhat homomorphic encryption. In ACM CCSW 2013. Berlin, Germany, 65-76.

[41] Samee Zahur, Mike Rosulek, and David Evans. April 26-30, 2015. Two Halves Make a Whole - Reducing Data Transfer in Garbled Circuits Using Half Gates. In EUROCRYPT 2015. Sofia, Bulgaria, 220-250. 